\title{
148 \\ MOMENTS AND CUMULANTS IN THE SPECIFICATION OF DISTRIBUTIONS
}

Author's Note (CMS 30.a)

The paper was intended as a compact summary of the more useful properties of those symmetric functions variously known as moments, semi-variants, cumulants, etc. To write it was a most enjoyable collaboration, since so much seemed worth doing. On rereading it now appears to be a great deal too compact. Without expanding the material to its "natural size" (a monograph textbook), I have in this edition ventured to ease the compression by inserting in a few places rather more explicit explanations. Table III has been added also for this edition. 


\title{
MOMENTS AND CUMULANTS IN THE SPECIFICATION OF DISTRIBUTIONS.
}

\author{
By E. A. Cornish and R. A. Fisher F. R.S.
}

1.

The very considerable statistical Iiterature which has grown up on the use of the moments of populations and samples, and on other quantities allied to these, is rendered confusing by variations in notation and terminology, and by the different aims which authors have had in view in using these quantities. The following notes aim at clarifying the subject by suggesting a uniform and consistent notation, specifying briefly the relations between the different quantities ordinarily used, and summarising the results which have been obtained.

The distribution of a variable quantity $x$ can be specified by means of a frequency function $f$, often termed the probability integral, specifying the total frequency in the population for which the variate is less than an assigned value $x$. For discontinuous distributions $f$ will be a step function, increasing discontinuously at the values of $x$ at which finite fractions of the total frequency are concentrated, and remaining constant between these values. For certain other distributions $f$ is continuous and differentiable so that $\frac{d f}{d x}$ represents the frequency density in the element of range $d x$, or, the ordinate of a frequency curve at this point. These are the two common cases, but it is also possible for $f$ to be continuous, but not differentiable, and so incapable of representation by a frequency curve.

\section{The Characteristic Function.}

In all cases we may define a function of a real variable $t$ in the form

$$
M(t)=\int_{-\infty}^{\infty} e^{i t x} d f,
$$

which is known as the characteristic function of the distribution. The absolute value of $M$ never exceeds unity for any real value of $t . M(t)$ and $M(-t)$ are equal, if real, and conjugate quantities, if complex. If, in the neigbourhood of $t=0, M$ can be expanded in a scries of powers of $t$, this series will be

or

$$
\sum_{r=0}^{\infty} \frac{(i t)^{r}}{r !} \int_{-\infty}^{\infty} x^{r} d f
$$

$$
\sum_{r=0}^{\infty} \frac{(i t)^{r}}{r !} \mu^{\prime}
$$

where $\mu^{\prime}$ is the $r$ th moment of the distribution of $x$ about the origin. The characteristic function may, therefore, be spoken of as the moment generating 
function. $\mu_{r}^{\prime}$ is, of course, the average value of $x^{r}$ and when this is finite, the characteristic function is differentiable $r$ times at the origin.

If $\mu_{1}^{\prime}$ is the mean, the factor $e^{i t x}$ may be resolved into the product

$$
e^{i t \mu_{1}^{\prime}} \cdot e^{i t}\left(x-\mu \mu_{1}\right)
$$

of which the first factor is constant, while the average value of the second factor gives a characteristic function referred to the mean of the distribution, and therefore formally expansible as a generating function of the moments about the mean. The relation between the moments about zero and the moments about the mean of the distribution may therefore be obtained by equating coefficients of powers of $t$ in the identity

$$
\begin{aligned}
& 1+\mu_{2} \frac{t^{2}}{2 !}+\mu_{3} \frac{t^{3}}{3 !}+\mu_{4} \frac{t^{4}}{4 !}+\ldots \ldots \\
& =e^{-\mu_{1} t}\left(1+\mu_{1}^{\prime} t+\mu_{2}^{\prime} \frac{t^{2}}{2 !}+\mu_{3}^{\prime} \frac{t^{3}}{3 !}+\mu_{4}^{\prime} \frac{t^{4}}{4 !}+\ldots \ldots\right)
\end{aligned}
$$

giving the series of relations

$$
\begin{aligned}
& \mu_{2}=\mu^{\prime}-\mu_{1}^{\prime}{ }^{2}, \\
& \mu_{3}=\mu_{3}^{\prime}-3 \mu_{2}^{\prime} \mu_{1}^{\prime}+2 \mu_{1}^{{ }^{3}}, \\
& \mu_{4}=\mu_{4}^{\prime}-4 \mu_{3}^{\prime} \mu_{1}^{\prime}+6 \mu_{2}^{\prime} \mu_{1}^{{ }^{2}}-3 \mu_{1}^{{ }_{1}{ }^{1}},
\end{aligned}
$$

by which the moments about the mean may be obtained from those about any other origin.

\section{The Cumulative Function.}

In studying the distributions of quantities compounded of ingredients, each distributed independently in a known distribution, Laplace was led to introduce a function known as the cumulative function, which is simply the logarithm of the characteristic function.

If $x$ is distributed in a distribution specified by the frequency element $d f_{1}$, and $y$ is independently distributed in a distribution specifed by the element $d f_{2}$, the frequency of the simultaneous occurrence of any particular pair of values $x$ and $y$ will be $d f_{1} d f_{2}$, and the characteristic function of the sum, $x+y$, will be

$$
\int_{-\infty}^{\infty} \int_{-\infty}^{\infty} e^{i t(x+y)} d f_{1} d f_{2},
$$

which is clearly the product of the characteristic functions of $x$ and $y$ separately. Consequently, if $K=\log M$, be written for the cumulative function, the cumulative function of $x+y$ is simply the sum of the cumulative functions of $x$ and $y$ separately. Evidently this relationship holds for any number of ingredients and is fundamental in the study of distributions of. compound quantities.

The identity of these functions for all values of $t$ carries with it the identity of their coefficients when $M$ and $K$ are expressible by power series. We are therefore led to recognise the coefficients of the expansion of $K$ in powers of $t$ as quantities of peculiar significance in the specification of the 
distribution. We shall call these quantities cumulants, denoted by $\kappa_{1}, \kappa_{2}, \kappa_{3} \ldots . .$, and defined by the identity

$$
\begin{aligned}
& \kappa_{1} t+\kappa_{2} \frac{t^{3}}{2 !}+\kappa_{3} \frac{t^{3}}{3 !}+\kappa_{4} \frac{t^{4}}{4 !}+\ldots \ldots \\
& =\log \left\{1+\mu_{1}^{\prime} t+\mu_{2}^{\prime} \frac{t^{2}}{2 !}+\mu_{3}^{\prime} \frac{t^{3}}{3 !}+\mu_{4}^{\prime} \frac{t^{4}}{4 !}+\ldots \ldots\right\},
\end{aligned}
$$

by which the moments about zero may be expressed in terms of cumulants, or vice versa, or by

$$
\begin{aligned}
& \kappa_{2} \frac{t^{2}}{2 !}+\kappa_{3} \frac{t^{3}}{3 !}+\kappa_{4} \frac{t^{4}}{4 !}+\ldots \ldots \\
& =\log \left\{1+\mu_{2} \frac{t^{2}}{2 !}+\mu_{3} \frac{t^{3}}{3 !}+\mu_{4} \frac{t^{4}}{4 !}+\ldots \ldots\right\}
\end{aligned}
$$

giving the corresponding relations with the moments about the mean. The latter are of great simplicity for

$$
\begin{aligned}
& \mu_{2}=\kappa_{2}, \\
& \mu_{3}=\kappa_{3}, \\
& \mu_{4}=\kappa_{4}+3 \kappa_{2}{ }^{2}, \\
& \mu_{5}=\kappa_{5}+10 \kappa_{3} \kappa_{2}
\end{aligned}
$$

and so on. The numerical coefficients may be written down at sight for the coefficient of $\kappa_{2}{ }^{2}$ is the number of ways in which 4 objects may be divided into two groups of 2 each, and that of $\kappa_{3} \kappa_{2}$ is the number of ways in which 5 objects may be divided into a group of 3 and a group of 2 . The same rule holds generally.

For, if

$$
\left(p_{1} \pi_{1} p_{2} \pi_{3} \ldots . .\right)
$$

stand for any partition of a number $r$, the coefficient of $\kappa_{p_{1}}^{\pi_{1}} \kappa_{p_{2}}^{\pi_{2}} \ldots$. . in the expansion of

$$
e^{\kappa_{2} \frac{t^{2}}{2 !}+\kappa_{3} \frac{t^{3}}{3 !}+\ldots \ldots}
$$

is seen to be

$$
\frac{t r}{\left(p_{1} !\right)^{\pi_{1}} \pi_{1} !\left(p_{2} !\right)^{\pi_{2}} \pi_{2} ! \ldots \ldots}
$$

so that the coefficient of $\kappa_{p_{1}}^{\pi_{1}} \kappa_{p_{2}}^{\pi_{2}} \ldots \ldots$ in $\mu_{r}$ is

$$
\frac{r !}{\left(p_{1} !\right)^{\pi_{1}} \pi_{1} !\left(p_{2} !\right)^{\pi_{2}} \pi_{2} ! \ldots \ldots}
$$

or the number of ways of distributing $r$ objects into undifferentiated receptacles $\pi_{1}$ containing $p_{1}$ each, $\pi_{2}$ containing $p_{2}$ each, and so on.

\section{Average effects of grouping.}

When, by reason of the limited accuracy of instrumental measurements, for convenience of record, or to simplify the calculations, variates are grouped so that to all values lying in the range $x-\frac{1}{2} h$ to $x+\frac{1}{2} h$ is assigned the conventional value $x$, the cumulants of the distribution will be somewhat affected. 
To any true value $\xi$, the process of grouping adds a grouping error $x-\xi$, where $x$ is the centre of the group in which $\xi$ falls. Knowing the group limits, we know also the actual error introduced for each possible value of $\xi$. With equal intervals this error will be a periodic function of $\xi$, and an exact study of the effects of grouping must involve the phase relationship between the group limits and any such characteristic of the population as its mean. With moderately fine grouping, the periodic corrections are, however, small; and it is often sufficiently accurate to consider only the average effects of grouping, when for a given grouping interval, $h$, the group limits are supposed to fall with equal frequency in any equal lengths in which the interval may be divided.

In this case the error of grouping is distributed with uniform frequency over the range from $-\frac{1}{2} h$ to $+\frac{1}{2} h$ so that its frequency distribution is

$$
d f=\frac{1}{h} \quad d x, \quad-\frac{1}{2} h<x<\frac{1}{2} h
$$

for all values of $\xi$ independently.

The average cumulants of the grouped distribution will therefore differ from those of the original ungrouped distribution by the cumulants of the grouping error.

The characteristic function is

$$
\begin{aligned}
M & =\frac{1}{h} \int_{-\frac{1}{2} e^{\frac{1}{2}} e^{i t x} d x} \\
& =\frac{2}{h t} \sin \frac{1}{2} h t . \\
& =1+\frac{h^{2}}{12} \frac{(i t)^{2}}{2 !}+\ldots \ldots+\frac{h^{2 r}}{2^{2 r}(2 r+1)} \frac{(i t)^{2 r}}{(2 r) !}+\ldots . .
\end{aligned}
$$

Hence

$$
K=\frac{h^{2}}{12} \frac{(i t)^{2}}{2 !}-\frac{h^{4}}{120} \frac{(i t)^{4}}{4 !}+\frac{h^{6}}{252} \frac{(i t)^{6}}{6 !}-\ldots . .
$$

Since, with group interval smaller than the standard deviation, the higher cumulants expressed in group units increase rapidly, the effects of grouping on them are extremely small, even when the second cumulant is materially affected.

The deduction of the coefficients $\frac{h^{2}}{12}-\frac{h^{4}}{120}, \frac{h^{6}}{252}, \ldots .$. from the cumulants estimated from grouped data is equivalent to Sheppard's adjustments of the moments.

\section{The symmetric function of a finite sample of observations of which the mean value is $\kappa_{w}$.}

It is easy to see that the condition that the mean value of a symmetric function of the observations shall be equal to one of the cumulants, or some function of the cumulants, for samples of all sizes, is sufficient to determine the symmetric function completely. This property was, however, long overlooked, and the series of statistics which afford unbiassed estimates of the cumulants was, in fact, only introduced in connection with a study of the sampling distributions of such estimates, which are found to be greatly simpli- 
fied, both in their form and their derivation, by using the appropriate series of statistics. Corresponding with any partition

$$
P=\left(p_{2} \pi_{1} p_{2} \pi_{2} \ldots . .\right), \quad S(\pi)=\rho, \quad S\left(p_{\pi}\right)=w,
$$

of the partible number $w$ there exists a monomial symmetric function of a sample of $n$ observations. If of the $n$ observations $\pi_{1}$ are chosen to be raised to the power of $p_{1}$; of the remainder we choose $\pi_{2}$ to be raised to the power of $p_{2}$, and so on, leaving $n-\rho$ observations not involved, the product of the powers of the chosen observations constitutes a typical term of the symmetric function. The number of similar terms that can be formed is

$$
\frac{n !}{\pi_{1} ! \pi_{2} ! \ldots \ldots(n-\rho) !}
$$

and the sum of these will be designated by the symbol $G(P)$.

Thus $G\left(2^{2} 1\right)$ stands for

$$
\frac{1}{2} \underset{r=1}{\stackrel{n}{S}} \stackrel{n}{S} \underset{s=1}{\stackrel{n}{S}}\left(x^{2} r_{r} x_{s}^{2} x_{t}\right)
$$

in which $r, s$ and $t$ may take any three different values from 1 to $n$. The factor $\frac{1}{2}$ is required since interchange of the values $r$ and $s$ leaves the monomial function unaltered.

Since the observations are independent, the mean value of any term is

so that

$$
\mu_{p_{1}}^{\pi_{1}} \mu_{p_{2}}^{\pi_{2}} \ldots \ldots
$$

But we know that

$$
\widetilde{G}(P)=\frac{n !}{\pi_{1} ! \pi_{2} ! \ldots \ldots(n-\rho) !} \mu_{p_{1}}^{\prime \pi_{1}} \quad \mu_{p_{2}}^{\prime \pi_{2}} \ldots \ldots .
$$

$$
\kappa_{w}=\sum_{\mu} \frac{(-)^{\rho-1}(\rho-1) ! w !}{\left(p_{1} !\right)^{\pi_{1}}\left(p_{2} !\right)^{\pi_{2}} \ldots \ldots \pi_{1} ! \pi_{2} ! \ldots \ldots} \mu_{p_{1}^{\prime}}^{\pi_{1}} \mu_{p_{2}^{\prime}}^{\pi_{2}} \ldots \ldots
$$

the summation extending over all partitions of $w$.

Hence to obtain a statistic $k_{w}$ such that $\bar{k}_{w}=\kappa_{w}$, it is sufficient to put

$$
k_{w}=\sum_{p} \frac{(-)^{p-1}(\rho-1) !}{n(n-1) \ldots \ldots(n-\rho+1)} \frac{w !}{\left(p_{1} !\right)^{\pi_{1}}\left(p_{2} !\right)^{\pi_{2}} \ldots \ldots} G(P) .
$$

The set of symmetric functions most easily calculated from the observations, at least if these are grouped, or have many repetitions of the same value, are the sums of powers $s_{q}=S(x q)$. where

From these, corresponding with any partition $Q$ of the partible number $w$,

$$
Q=\left(q_{1}^{x_{1}} q_{2}{ }^{x_{2}} \ldots \ldots\right)
$$

it is easy to construct the symmetric function

$$
S(Q)=s_{q_{1}}^{\chi_{1}} s_{q_{2}}^{\chi_{2}} \ldots \ldots
$$

To express $G(P)$ in terms of $S(Q)$ we require the bipartitional function Gs $(P, Q)$ defined by the identity

$$
G(P)=\sum_{Q} G s(P, Q) S(Q)
$$

Gs is found to be an integer divided by $\pi_{1} ! \pi_{2} ! \ldots \ldots$; for values of $w$ from 2 to 6 the values of $\pi_{1} ! \pi_{2} ! \ldots \ldots G s(P, Q)$ are tabulated below. 


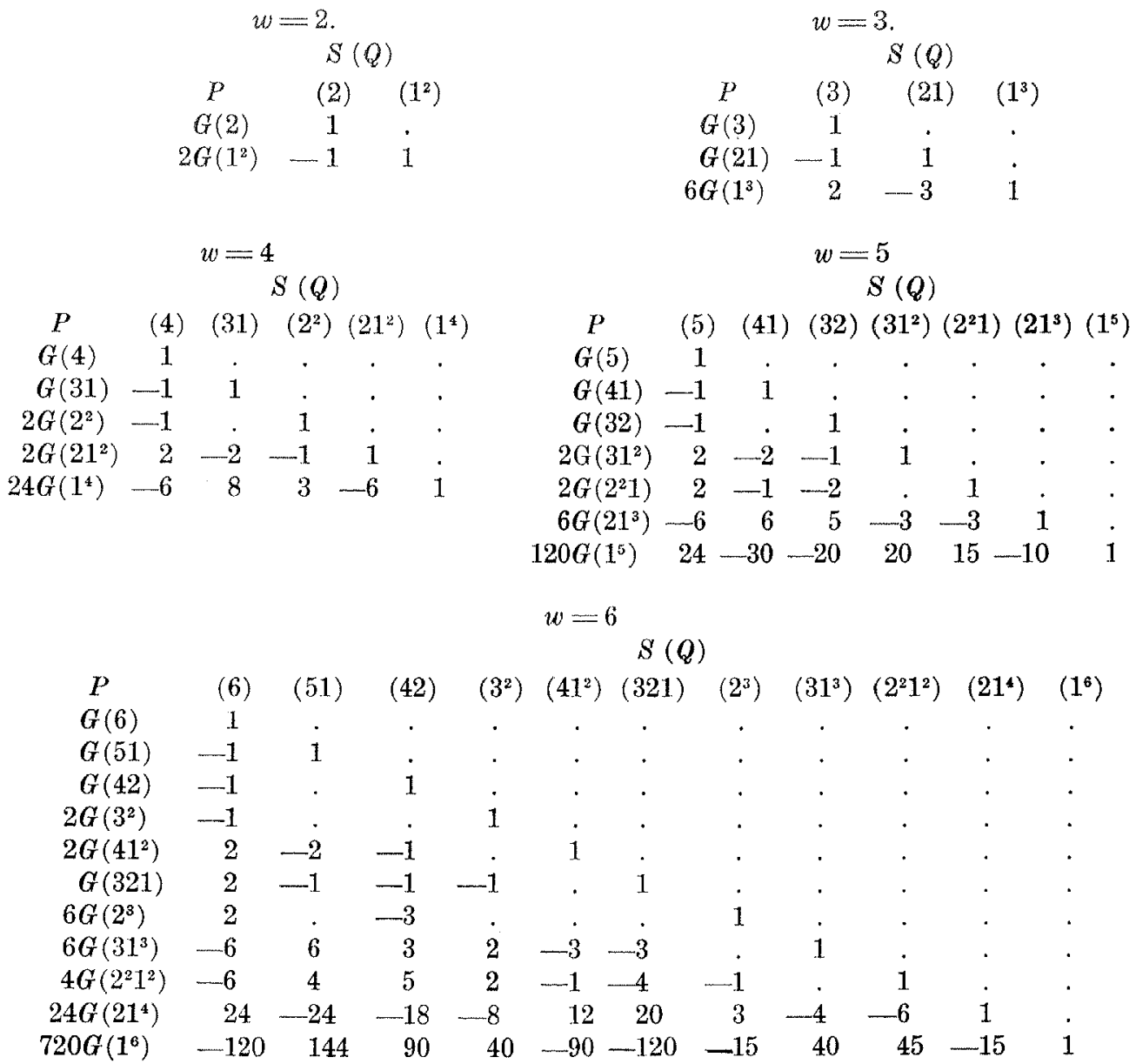

In the expression for $k_{w}$ the coefficient of $G(P)$ for partitions of a fixed number of parts $\rho$, is proportional to

$$
\frac{w !}{\left(p_{1} !\right)^{\pi_{1}}\left(p_{2} !\right)^{\pi_{2}} \ldots \ldots}=\pi_{1} ! \pi_{2} ! \ldots \ldots a(P)
$$

where $a(P)$ is the elementary partitional function

$$
\frac{w !}{\left(p_{1} !\right)^{\pi_{1}} \pi_{1} !\left(p_{2} !\right)^{\pi_{2}} \pi_{2} ! \ldots \ldots} .
$$

We may therefore use the property of the function $G s(P, Q)$, namely that

$$
\sum_{P / P}^{\Sigma} \pi_{1} ! \pi_{2} ! \ldots \ldots a(P) G s(P, Q)
$$

is $a(Q)$ times the coefficient of $x^{p}$ in the product, for all parts $q$ of $Q$, of the polynomials

$$
F^{\prime}(q)=x \sum_{r=0}(-x \Delta) r(1 q-1)
$$


For the smaller values of $q$ we have

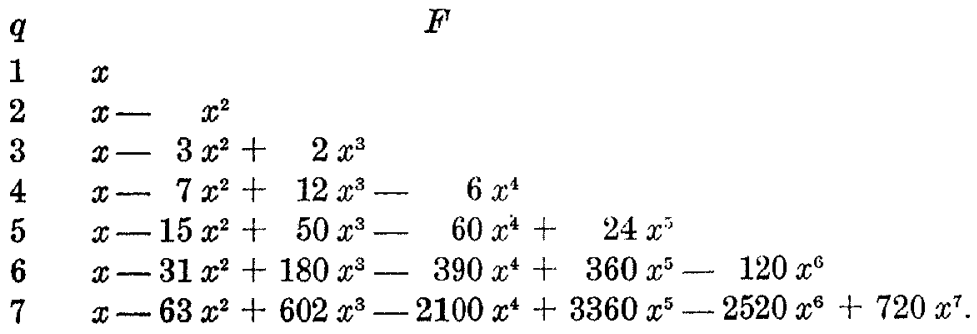

The expression for $k_{w}$ thus reduces to

$$
k_{v}=\Sigma_{Q}\left\{\sum_{\rho} \frac{(-)^{p-1}(\rho-1) !}{n(n-1) \ldots \ldots(n-\rho+1)} u_{f} a(Q) S(Q)\right\}
$$

where $u_{\rho}$ is given by

$$
F^{\chi_{1}}\left(q_{1}\right) F^{\chi_{2}}\left(q_{2}\right) \ldots \ldots=\mathrm{\Sigma} u_{p} x_{p} .
$$

The process of simplification may be illustrated by determining the coefficient of $s_{3} s_{2}^{2}$ in $k_{7}$. We have $Q=\left(32^{2}\right), a(Q)=105, F^{\prime}(3) F^{2}(2)=x^{3}-5 x^{4}+9 x^{5}-7 x^{6}+2 x^{7}=\Sigma u_{\rho} x^{p}$, so that the coefficient of $s_{3} s_{2}{ }^{2}$ is

$$
\begin{gathered}
a(Q) \sum_{\rho} \frac{(\rho-1) !(-)^{\rho-1}}{n(n-1) \ldots \ldots(n-\rho+1)} u_{\rho} \\
=105\left(\frac{2 !}{n(n-1)(n-2)} 1+\frac{3 !}{n(n-1)(n-2)(n-3)} 5\right. \\
+\frac{4 !}{n(n-1)(n-2)(n-3)(n-4)} 9+\frac{5 !}{n(n-1)(n-2)(n-3)(n-4)(n-5)} 7 \\
\left.+\frac{6 !}{n(n-1)(n-2)(n-3)(n-4)(n-5)(n-6)} 2\right)=\frac{210 n}{(n-3)(n-4)(n-5)(n-6)}
\end{gathered}
$$

5. Transformation of the characteristic function.

If $\xi$ is any function of $x$ capable of expansion in a power series

$$
\xi(x)=a_{0}+a_{1} x+a_{2} x^{2}+\ldots \ldots
$$

then the characteristic function of $\xi$, is the average value of $e^{i \tau \xi}$.

The coefficient of $(i \tau) r / r$ ! is the average value of

$$
\left(a_{0}+a_{1} x+a_{2} x^{2}+\ldots \ldots\right)^{r}
$$

or, if the characteristic function $M(t)$ is differentiable

$$
\left\{a_{0}+a_{1} \frac{d}{d(i t)}+a_{2} \frac{d^{2}}{d(i t)^{2}}+\ldots \ldots\right\}^{r} M(t) .
$$

Hence, the characteristic function of any function $\xi(x)$ may be expressed in terms of that of $x$ in the form

$$
M_{\xi}(\tau)=e^{-i \tau \xi\{r l d(i t)\}} M_{x}(t), \text { when } t=0 .
$$

\section{The operational properties of the cumulants.}

If the element of frequency is $y d x$, and $y$ and its differential coefficients vanish at the limits of the range

* See Author's Addendum at end of paper (page 28). 


$$
\begin{aligned}
& \int e^{i t x}\left(\frac{d r}{d x^{r}} y\right) d x=\left[e^{i l x} \frac{d^{r-1}}{d x^{r-1}} y\right]-i t \int e^{i t x}\left(\frac{d^{r-1}}{d x^{r}-1} y\right) d x \\
& =-i t \int e^{i t x}\left(\frac{d^{r-1}}{d x^{r}-1} y\right) d x \\
& =(-i t)^{r} M \text {. }
\end{aligned}
$$

Hence

$$
\int e^{i t x} e^{a_{q}\left(-\frac{d}{d x}\right)^{q}} y d x=M e^{a_{q}(i t)^{q}}
$$

It thus appears that the cumulative function of the distribution

differs from that of

$$
d f=e^{a} q\left(-\frac{d}{d x}\right)^{q} y d x
$$

by

or that the operator

$$
\begin{aligned}
& d f=y d x \\
& a_{q}(i t)^{\eta}
\end{aligned}
$$

$$
e^{\frac{a q}{q !}\left(-\frac{d}{d x}\right)^{q}}
$$

merely increases the $q^{\text {th }}$ eumulant by $a_{q}$.

The action of the operator $e^{-a_{1} \frac{d}{d x}}$ merely transforms a function $f(x)$ into $f\left(x-a_{1}\right)$; when acting on a frequency function, it thus simply increases the mean by $a_{1}$, leaving the distribution otherwise unchanged. Similarly it appears that

$$
e^{\frac{a_{3}}{2 !} \frac{d^{2}}{d x^{2}}}
$$

simply increases the variance by $a_{2}$, leaving the mean and other cumulants unchanged, as would be done by seattering each element of frequency in a normal distribution with variance $a_{2}$. Similarly, the other operators of the form

$$
e^{\frac{a_{q}}{q !}\left(-\frac{d}{d x}\right)^{q}}
$$

may be used to adjust any of the other cumulants to desired values.

\section{The probability integral of a distribution having given cumulants.}

Since the frequency element of the distribution of a variable $\xi$ having given cumulants $\kappa_{1}, \kappa_{2}, \kappa_{3}, \ldots .$. can be represented formally by

$$
d p=\exp \left\{-\left(\kappa_{1}-m\right) \frac{d}{d \xi}+\frac{1}{2}\left(\kappa_{2}-v\right) \frac{d^{2}}{d \xi^{2}}-\frac{1}{6} \kappa_{3} \frac{d^{3}}{d \xi^{3}}+\ldots \ldots\right\} \frac{1}{\sqrt{2 \pi v}} e^{\frac{-(\xi-m)^{2}}{2 v}} d \xi
$$

where $m$ and $v$ are the mean and variance of any normal distribution chosen for convenience, we may use this choice to simplify the determination of the probability integral.

Sometimes the exact values of $\kappa_{1}$ and $\kappa_{2}$ may be used; in this case the expression will only involve the higher cumulants $\kappa_{3}, \kappa_{4} \ldots \ldots$. More frequently the successive cumulants are expressed in power series of the reciprocal of some number $n$, so that the order of magnitude of $\kappa_{r}$ is that of $n^{-(r-1)}$. 
If $m$ and $v$ be chosen to agree with the leading terms of the series for $\kappa_{1}$ and $\kappa_{2}$, the ratio of $\kappa_{r}$ to $v^{\frac{1}{2} r}$ will be of the order of $n^{-\left(\frac{1}{2} r-1\right)}$, when $r$ exceeds 2, and the expansion takes the form

$d p=\exp \left\{-a v^{\frac{1}{2}} \frac{d}{d \xi}+\frac{1}{2} b v \frac{d^{2}}{d \xi^{2}}-\frac{1}{6} c v^{\frac{3}{2}} \frac{d^{3}}{d \xi^{3}}+\ldots \ldots\left\{\frac{1}{\sqrt{2 \pi v}} e^{\frac{-(\xi-m)^{2}}{2 v}} d \xi\right.\right.$

where $a$ and $c$ are of order $n^{-\frac{1}{2}}, b$ and $d$ of order $n^{-1}, e$ of order $n^{-\frac{3}{2}}$, $f$ of order $n^{-2}$, and so on.

Expanding the operator and integrating, we have for the frequency less than $m+\xi v^{\frac{1}{2}}$, the expansion, of which the first four adjustment terms have been retained,

$$
\begin{gathered}
p \\
-z\left(a+\frac{1}{6} c \xi_{2}\right) \\
+z\left(\frac{1}{2} a^{2} \xi_{1}+\frac{1}{2} b \xi_{1}+\frac{1}{6} a c \xi_{3}+\frac{1}{24} d \xi_{3}+\frac{1}{72} c^{2} \xi_{5}\right) \\
-z\left(\frac{1}{6} a^{3} \xi_{2}+\frac{1}{2} a b \xi_{2}+\frac{1}{12} a^{2} c \xi_{4}+\frac{1}{12} b c \xi_{4}+\frac{1}{24} a d \xi_{4}+\frac{1}{120} e \xi_{4}+\frac{1}{72} a c^{2} \xi_{5}\right. \\
\left.+\frac{1}{144} c d \xi_{5}+\frac{1}{1296} c^{3} \xi_{8}\right) \\
+z\left(\frac{1}{24} a^{4} \xi_{3}+\frac{1}{8} b^{2} \xi_{3}+\frac{1}{4} a^{2} b \xi_{3}+\frac{1}{36} a^{3} c \xi_{5}+\frac{1}{12} a b c \xi_{5}+\frac{1}{48} a^{2} d \xi_{5}+\frac{1}{48} b d \xi_{5}\right. \\
+\frac{1}{120} a e \xi_{5}+\frac{1}{720} f \xi_{5}+\frac{1}{144} a^{2} c^{2} \xi_{7}+\frac{1}{144} b c^{2} \xi_{7}+\frac{1}{1152} d^{2} \xi_{7}+\frac{1}{144} a c d \xi_{7} \\
\left.+\frac{1}{720} c e \xi_{7}+\frac{1}{1296} a c^{3} \xi_{9}+\frac{1}{1728} c^{2} d \xi_{9}+\frac{1}{31104} c^{4} \xi_{11}\right)
\end{gathered}
$$

where

$$
\begin{gathered}
z=\frac{1}{\sqrt{2 \pi}} e^{-\frac{1}{2} \xi^{2}}, \\
p=\int_{-\infty}^{\xi} z d \xi
\end{gathered}
$$

and $\xi_{r}$ is the Hermite polynomial given by

or in full,

$$
\frac{d r}{d \xi^{r}} \quad z=\xi_{r} z
$$

$$
\begin{array}{rlrl}
\xi_{1} & =-\xi & \xi_{2} & =\xi^{2}-1 \\
\xi_{3} & =-\xi^{3}+3 \xi & \xi_{4} & =\xi^{4}-6 \xi^{2}+3 \\
\xi_{5} & =-\xi^{5}+10 \xi^{3}-15 \xi & \xi_{6} & =\xi^{8}-15 \xi^{4}+45 \xi^{2}-15 \\
\xi_{7} & =-\xi^{7}+21 \xi^{5}-105 \xi^{3}+105 \xi & \xi_{8} & =\xi^{8}-28 \xi^{6}+210 \xi^{4}-420 \xi^{2}+105 \\
\xi_{a} & =-\xi^{3}+36 \xi^{T}-378 \xi^{5}+1260 \xi^{3}-945 \xi & \xi_{10} & =\xi^{10}-45 \xi^{8}+630 \xi^{6}-3150 \xi^{4}+4725 \xi^{2}-945 \\
\xi_{11}=-\xi^{11}+55 \xi^{9}-990 \xi^{7}+6930 \xi^{6}-17325 \xi^{3}+10395 \xi .
\end{array}
$$


8. The expansion for the abscissa corresponding to any given level of probability.

Although it is sometimes of interest to work out the actual value of the probability corresponding with a given deviation, it is of much more general utility to know the values of the deviates corresponding with the assigned levels of probability. If now we write $x$ for the normal deviate having the same probability integral, the difference $\xi-x$ may be found by equating the expression above for the probability to

$$
p-(\xi-x) z+\frac{1}{2}(\xi-x)^{2} \xi_{1} z-\frac{1}{6}(\xi-x)^{3} \xi_{2} z+\frac{1}{24}(\xi-x)^{4} \xi_{3} z .
$$

By equating terms of each order of magnitude in succession, as in the inversion of power series, we find the polynomials are much simplified, giving $\xi-x=a+\frac{1}{6} c\left(\xi^{2}-1\right)$

$$
\begin{aligned}
& +\frac{1}{2} b \xi-\frac{1}{3} a c \xi+\frac{1}{24} d\left(\xi^{3}-3 \xi\right)-\frac{1}{36} c^{2}\left(4 \xi^{3}-7 \xi\right) \\
& -\frac{1}{2} a b+\frac{1}{6} a^{2} c-\frac{1}{12} b c\left(5 \xi^{2}-3\right)-\frac{1}{8} a b\left(\xi^{2}-1\right) \\
& -+\frac{1}{120} e\left(\xi^{4}-6 \xi^{2}+3\right)+\frac{1}{36} a c^{2}\left(12 \xi^{2}-7\right) \\
& -\frac{1}{144} c d\left(11 \xi^{4}-42 \xi^{2}+15\right)+\frac{1}{648} c^{3}\left(69 \xi^{4}-187 \xi^{2}+52\right) \\
& -\frac{3}{8} b^{2} \xi+\frac{5}{6} a b c \xi+\frac{1}{8} a^{2} d \xi-\frac{1}{48} b d\left(7 \xi^{3}-15 \xi\right)-\frac{1}{30} a e\left(\xi^{3}-\xi\right) \\
& +\frac{1}{720} f\left(\xi^{5}-10 \xi^{3}+15 \xi\right)-\frac{1}{3} a^{2} c^{2} \xi+\frac{1}{72} b c^{2}\left(36 \xi^{3}-49 \xi\right) \\
& -\frac{1}{384} d^{2}\left(5 \xi^{5}-32 \xi^{3}+35 \xi\right)+\frac{1}{36} a c d\left(11 \xi^{3}-21 \xi\right) \\
& +\frac{1}{360} c e\left(7 \xi^{5}-48 \xi^{3}+51 \xi\right)-\frac{1}{324} a c^{3}\left(138 \xi^{3}-187 \xi\right) \\
& +\frac{1}{864} c^{2} d\left(111 \xi^{5}-547 \xi^{3}+456 \xi\right)-\frac{1}{7776} c^{4}\left(948 \xi^{\xi}-3628 \xi^{3}+2473 \xi\right)
\end{aligned}
$$

In these expressions it should be noticed that the polynomials involved are in the deviate $\xi$. It is in many ways more convenient to use instead polynomials in the normal deviate $x$, corresponding to the probability required. This involves an awkward substitution, which may, perhaps, best be carried out by observing that if

$$
\xi-x=f(\xi)=f\{x+(\xi-x)\},
$$

then it may be written to the required degree of approximation as

$$
\begin{gathered}
f(x)+f(x) f^{\prime}(x)+f(x) f^{\prime 2}(x)+\frac{1}{2} f^{2}(x) f^{\prime \prime}(x) \\
+f(x) f^{\prime 3}(x)+\frac{3}{2} f^{2}(x) f^{\prime}(x) f^{\prime \prime}(x)+\frac{1}{6} f^{3}(x) f^{\prime \prime \prime}(x) . \\
\text { or } \quad f(x)+\frac{1}{2} \frac{d}{d x} f^{\prime 2}(x)+\frac{1}{6} \frac{d^{2}}{d x^{2}} f^{3}(x)+\frac{1}{24} \frac{d^{3}}{d x^{3}} f^{4}(x)+\ldots,
\end{gathered}
$$


In the result of this substitution all but one of the terms involving $a$ must be eliminated, since a change of the mean leaving all other cumulants unchanged, changes all points of fixed probability by the same amount. The adjustment to the normal deviate $x$ having the required probability integral is:

$$
\begin{gathered}
a+\frac{1}{6} c\left(x^{2}-1\right) \\
+\frac{1}{2} b x+\frac{1}{24} d\left(x^{3}-3 x\right)-\frac{1}{36} c^{2}\left(2 x^{3}-5 x\right) \\
-\frac{1}{6} b c\left(x^{2}-1\right)+\frac{1}{120} e\left(x^{4}-6 x^{2}+3\right)-\frac{1}{24} c d\left(x^{4}-5 x^{2}+2\right) \\
+\frac{1}{324} c^{3}\left(12 x^{4}-53 x^{2}+17\right) \\
-\frac{1}{8} b^{2} x-\frac{1}{16} b d\left(x^{3}-3 x\right)+\frac{1}{720} f\left(x^{5}-10 x^{3}+15 x\right) \\
+\frac{1}{72} b c^{2}\left(10 x^{3}-25 x\right)-\frac{1}{384} d^{2}\left(3 x^{5}-24 x^{3}+29 x\right) \\
-\frac{1}{180} c e\left(2 x^{5}-17 x^{3}+21 x\right)+\frac{1}{288} c^{2} d\left(14 x^{5}-103 x^{3}+107 x\right) \\
-\frac{1}{7776} c^{4}\left(252 x^{5}-1688 x^{3}+1511 x\right) .
\end{gathered}
$$

For numerical work the polynomials in $x$ required may very easily be tabulated for chosen levels of probability (Table I). In Table II are given the numerical values of the first five Hermite Polynomials over the range of levels of probability chosen for Table $I$.

8. 1. The cumulants of the distribution of the test of significance, $z$, and the approximate values for different levels of significance.

For the purpose of obtaining its cumulants, we may write $z$, which is half the logarithm of the ratio of two estimated variances, in the form

$$
z=\frac{1}{2} \log \chi_{1}^{2} / n_{1}-\frac{1}{2} \log \chi_{2}^{2} / n_{2} \text {. }
$$

Now the distribution of $\chi_{1}$ is given by

$$
d f=\frac{1}{\frac{n_{1}-2}{2}} ! e^{-\frac{1}{2} x_{1}^{2}}\left(\frac{x_{1}^{2}}{2}\right)^{\frac{n_{1}-2}{2}} d\left(\frac{x_{1}^{2}}{2}\right)
$$

so that the mean value of $\exp \left\{\frac{1}{2} i t \log \chi_{1}{ }^{2} / n_{1}\right\}$

$$
=\exp \left\{\frac{1}{2} i t \log \left(\frac{1}{2} x_{1}^{2}\right)-\frac{1}{2} i t \log \frac{1}{2} n_{1}\right\}
$$

is

$$
\frac{\frac{n_{1}+i t-2}{2} !}{\frac{n_{1}-2}{2} !} \exp \left(-\frac{1}{2} i t \log \frac{1}{2} n_{1}\right)
$$

whence the cumulative function of $z$ is seen to be

$$
\begin{aligned}
& K=\log \frac{n_{1}+i t-2}{2} !-\log \frac{n_{1}-2}{2} !-\frac{1}{2} i t \log \frac{1}{2} n_{1} \\
& -\left(\log \frac{n_{2}+i t-2}{2} !-\log \frac{n_{2}-2}{2} !-\frac{1}{2} i t \log \frac{1}{2} n_{2}\right) .
\end{aligned}
$$




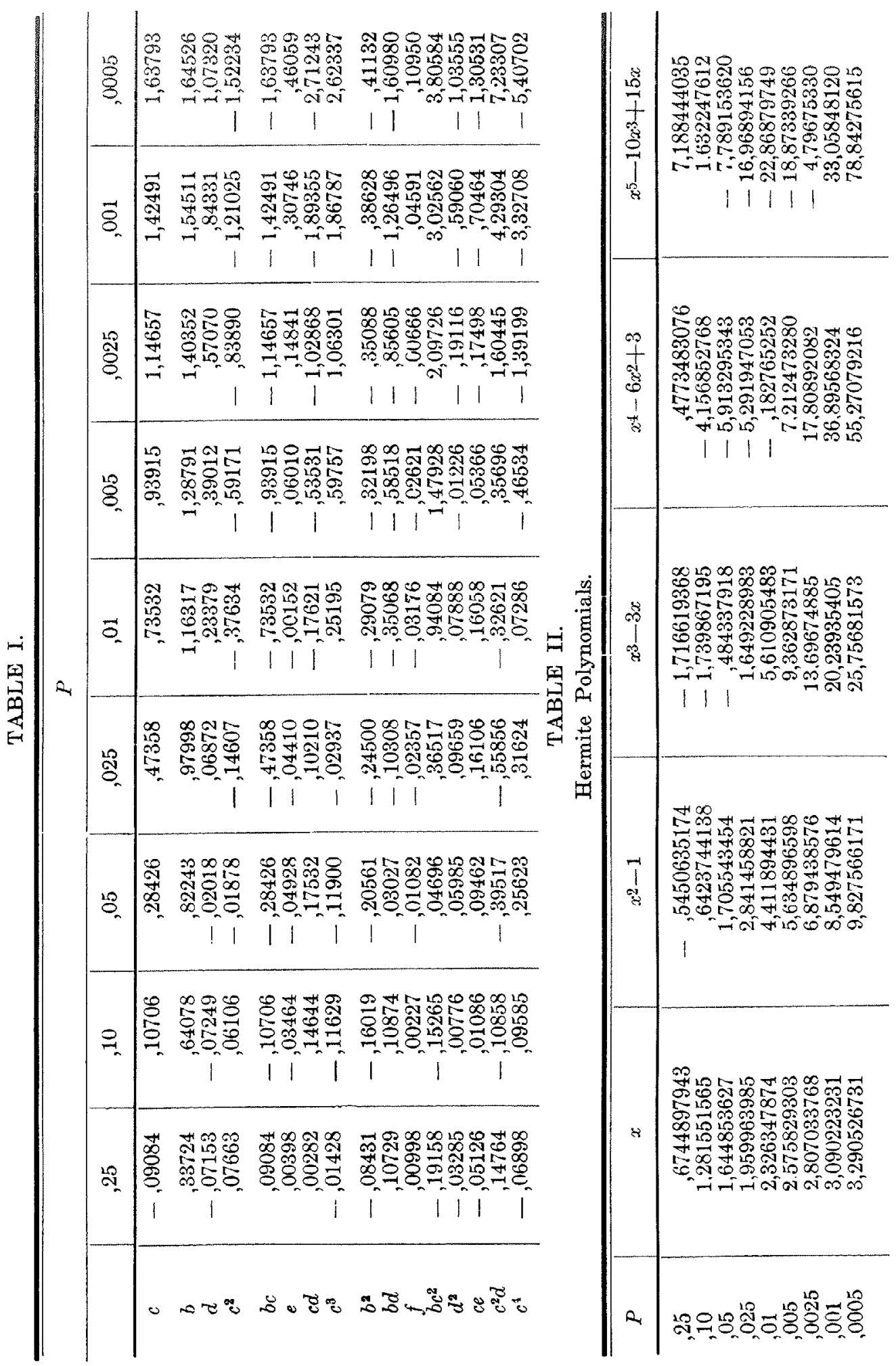


The expansion of $K$ in powers of it, riay therefore be found from the differential coefficients with respect to $n$ of

$$
\log \frac{n-2}{2} ! \text {. }
$$

Now with sufficient approximation

$$
\log \frac{n-2}{2} !=\frac{1}{2}(n-1) \log \frac{1}{2} n-\frac{1}{2} n+\frac{1}{2} \log (2 \pi)+\frac{1}{6 n},
$$

and its successive differential coefficients are:

$$
\begin{aligned}
& \frac{1}{2} \log \frac{1}{2} n-\frac{1}{2 n}-\frac{1}{6 n^{2}} \\
& \frac{1}{2 n}+\frac{1}{2 n^{2}}+\frac{1}{3 n^{3}} \\
& -\frac{1}{2 n^{2}}-\frac{1}{n^{3}} \\
& \frac{1}{n^{3}}+\frac{3}{n^{4}} \\
& -\frac{3}{n^{4}} \\
& \frac{12}{n^{5}}
\end{aligned}
$$

From these, writing $r_{1}$ and $r_{2}$ for the reciprocals of $n_{1}$ and $n_{2}$ respectively, we may obtain the cumulants

$$
\begin{aligned}
& \kappa_{1}=-\frac{1}{2}\left(r_{1}-r_{2}\right)-\frac{1}{6}\left(r_{1}^{2}-r_{2}^{2}\right), \\
& \kappa_{2}=\frac{1}{2}\left(r_{1}+r_{2}\right)+\frac{1}{2}\left(r_{1}^{2}+r_{2}^{2}\right)+\frac{1}{3}\left(r_{1}^{3}+r_{2}^{3}\right), \\
& \kappa_{3}=-\frac{1}{2}\left(r_{1}^{2}-r_{2}^{2}\right)-\left(r_{1}^{3}-r_{2}^{3}\right), \\
& \kappa_{4}=\left(r_{1}^{3}+r_{2}^{3}\right)+3\left(r_{1}^{4}+r_{2}^{4}\right), \\
& \kappa_{5}=-3\left(r_{1}^{4}-r_{2}^{4}\right), \\
& \kappa_{6}=12\left(r_{1}^{5}+r_{2}^{5}\right) .
\end{aligned}
$$

If we write $\sigma$ for the sum $\left(r_{1}+r_{2}\right)$ and $\delta$ for the difference $\left(r_{1}-r_{2}\right)$ of these reciprocals, and if we choose

then

$$
m=0, \quad v=\frac{1}{2} \sigma
$$

$$
\begin{aligned}
& a=-\sqrt{\frac{2}{\sigma}}\left(\frac{1}{2} \delta+\frac{1}{6} \delta \sigma\right), \\
& b=\frac{1}{2}\left(\sigma+\frac{\delta^{2}}{\sigma}\right)+\frac{1}{6}\left(\sigma^{2}+3 \delta^{2}\right), \\
& c=-\sqrt{\frac{2}{\sigma}}\left\{\delta+\frac{1}{2}\left(3 \delta \sigma+\frac{\delta^{3}}{\sigma}\right)\right\}, \\
& d=\left(\sigma+\frac{3 \delta^{2}}{\sigma}\right)+\frac{3}{2}\left(\sigma^{2}+6 \delta^{2}+\frac{\delta^{4}}{\sigma^{2}}\right), \\
& e=-\sqrt{\frac{2}{\sigma}}\left\{6 \delta\left(\sigma+\frac{\delta^{2}}{\sigma}\right)\right\}, \\
& f=6\left(\sigma^{2}+10 \delta^{2}+\frac{5 \delta^{4}}{\sigma^{2}}\right) .
\end{aligned}
$$


For axy special values of $n_{1}$ and $n_{2}$ the six cumulant adjustments can be evaluated numerically, and the values substituted in the general formula given above. It is, however, also of interest to make the substitution algebraically and obtain the general form of the $z$ values at different levels of significance in terms of $\sigma$ and $\delta$. We then have

$$
\begin{gathered}
x \sqrt{\frac{1}{2} \sigma} \\
-\frac{1}{6} \delta\left(x^{2}+2\right) \\
+V \overline{\frac{1}{2} \sigma}\left\{\frac{\sigma}{24}\left(x^{3}+3 x\right)+\frac{1}{72} \frac{\delta^{2}}{\sigma}\left(x^{3}+11 x\right)\right\} \\
+V \frac{\delta \sigma}{120}\left(x^{4}+9 x^{2}+8\right)+\frac{\delta^{3}}{3240 \sigma}\left(3 x^{4}+7 x^{2}-16\right) \\
+\frac{\sigma^{2}}{1920}\left(x^{5}+20 x^{3}+15 x\right)+\frac{\delta^{4}}{2880}\left(x^{5}+44 x^{3}+183 x\right) \\
\left.+\frac{\delta^{4}}{155520 \sigma^{2}}\left(9 x^{5}-284 x^{3}-1513 x\right)\right\}
\end{gathered}
$$

Example.

When $n_{1}=24$ and $n_{2}=60$ the $5 \%$ value of $z$ is .26534844 . The value obtained from the first approximation and the four correction terms above is $.28091224-.01960643+(.00389559+.00057292)-(.00048210-.00000206)$

\begin{tabular}{|c|c|c|c|}
\hline Degree & $\begin{array}{l}\text { Successive } \\
\text { Terms }\end{array}$ & $\begin{array}{l}\text { Successive } \\
\text { Totals }\end{array}$ & $\begin{array}{c}\text { Successive } \\
\text { Errors }\end{array}$ \\
\hline 0 & .28091224 & .28091224 & +.01556380 \\
\hline 1 & -1960643 & .26130581 & $-\quad 404263$ \\
\hline 2 & $+\quad 446851$ & .26577432 & 42588 \\
\hline 3 & 48004 & .26529428 & 5416 \\
\hline 4 & 5645 & .26535073 & 229 \\
\hline
\end{tabular}

$$
+(.00003805+.00001886-.00000046)
$$

$=26535073$.

The successive errors in this case diminish progressively, with alternating sign, as shown in Table III, (CMS 30.14a).

\section{TABLE III}


Résumé: Los cumulants d'une distribution sont definis ot leurş rolations avee les moments ordinaires sont indiquées. On insiste sur l'importance de ces fonotions dans la description des distributions.

La fonction symétrique d'un échantillon fini d'observations dont la valezr moyenne est égale au cumulant correspondant de la population est défini et une expression convenable pour son évaluation est développée en termes de sommes de puissances dess observations de l'échantillon.

Les propriétés opératives des cumulants sont discutés et le développement de l'intégrale des probabilités d'une distribution ayant des cumulants donné en dérivé. Ce développement est transformé en une expression plus cotmmode, qui donne la valeur des déviations correspondant à un niveau donné de probabilité, en fonction de la déviation normale ayant la même intégrale des probabilités. Les valeurs numériques des polynômes dans la déviation normale considérés dans le dernier développement sont calculés pour certains niveaux choisis de probabilité. De plus, les valeurs numériques des 5 premiers polynómes d'Hermite sont donnés pour les mêmes niveaux de probabilité. Un exemple de l'emploi des formules développées est donné par le calcul de la valeur approximative de $z$ pour le niveau de probabilité de 5 p.cent.

Author's Addendum (CMS 30.7a) - see page 313.

Since of the partitions of 7 only four are free from unitary parts, the complete expression for $k_{7}$ in terms of sums of powers, $s_{q}$, of deviations from the mean, may be quickly found to be

$$
\begin{aligned}
\frac{n}{(n-2)(n-3)(n-4)(n-5)(n-6)}\left\{n\left(n^{3}+42 n^{2}+119 n-42\right) \frac{s_{7}}{n-1}\right. \\
\left.-21\left(n^{2}+13 n-18\right) s_{6} s_{2}-35\left(n^{2}+n+6\right) s_{4} s_{3}+210(n-2) s_{3} s_{2}^{2}\right\} .
\end{aligned}
$$

If deviations are not measured from the mean of the sample, these terms are unchanged, but the terms corresponding with the eleven partitions which involve unitary parts will also be required. Full expression for the lower values of $k$ were given in Moments and Product Moments of Sampling Distributions, Proc. London Math. Soc., Ser. 2, 30, pt. 3 (1928). 